\title{
Robot pathfinding with obstacle avoidance capabilities in a static indoor environment via TOR iterative method using harmonic potentials
}

\author{
A'Qilah Ahmad Dahalan ${ }^{1 *}$, Azali Saudi², and Jumat Sulaiman ${ }^{3}$ \\ ${ }^{1}$ Centre for Defence Foundation Studies, National Defence University of Malaysia, Kuala Lumpur, \\ Malaysia \\ ${ }^{2}$ Faculty of Computing \& Informatics, Universiti Malaysia Sabah, Kota Kinabalu, Malaysia \\ ${ }^{3}$ Faculty of Science \& Natural Resources, Universiti Malaysia Sabah, Kota Kinabalu, Malaysia
}

\begin{abstract}
Mobile robots are always in a state where they have to find a collision-free path in their environment from start to the target point. This study tries to solve the problem of mobile robot iteratively by using a numerical technique. It is based on potential field technique that was modelled using the Laplace's equation to restrain the creation of a potential functions across regions in the mobile robot's configuration space. The gradient formed by the potential field is then used to generate a path for the robot to advance through. The present paper proposes a Two-Parameter Over-Relaxation (TOR) iterative method that is used to solve Laplace's equation for obtaining the potential field that is then utilized for finding path of the robot, thus solving the robot pathfinding problem. The experiment indicates that it is capable of producing a smooth path between the starting and target points through the use of a finite-difference technique. Furthermore, the simulation results show that this numerical approach executes quicker and provides a smoother trail than to the previous works, that includes Successive Over-Relaxation (SOR) and Accelerated OverRelaxation (AOR) methods.
\end{abstract}

\section{Introduction}

Pathfinding or navigation problem plays an important role in autonomous mobile robots. To construct a genuinely self-directed mobile robot, it must be able to design a passage from initial to the final configurations efficiently and reliably without interfering with obstacles in between. Competent algorithms for unraveling this kind of problem have substantial applications in fields such as computer animation, industrial robotics, automated surveillance, and drug design. Hence, it is of no surprise that over the last two decades, the research activities in this field have gradually increased.

This paper aims to simulate a point-robot pathfinding in the configuration space using numerical potential functions based on heat transfer theory. This heat transfer model crafts an environment free from local minima as well as beneficial for the control of robot

*Corresponding author: a.qilah@upnm.edu.my 
navigation. The problem of heat transfer in this study is posed as Laplace's equation. Laplace's equation solutions better recognized as harmonic functions, mathematically describe the temperature values for the path generation model in the configuration space. There have been different approaches used to obtain harmonic functions but, by reason of the obtainability of fast processing machines and their elegance and competence in resolving the problem, the most general approach is via numerical techniques. Within this study, three experiments were carried out to investigate the efficiency of the accelerated iterative method used in producing paths of a mobile robot for multi-size environments.

Connolly and Gruppen [1] had shown in their pioneering studies that harmonic functions possess many useful properties in the applications of robotic. Meanwhile, Khatib [2] used potential robot pathfinding functions, in which each obstacle produces force repellents while the targets exert an attractive force. However, the core shortcoming of these potential fields was that they were suffering on the generation of local minima.

A global approach that uses solutions of Laplace's equations to produce a smooth trail, which the potential fields were identified in a global manner throughout the area, had been established [3, 4]. Previous works [5, 6] showed over-relaxation methods performed much quicker compared to standard Gauss-Seidel or Jacobi iterative methods. A number of other approaches in solving the problem of pathfinding had also been suggested. Willms and Simon [7] indicated a system that employed distance transform technique. Then, Jan et al. [8] led studies on the exploitation of the routing algorithm for geometry maze. Whereas Bhattacharya and Gavrilova [9] developed a Voronoi Diagram based approach. In addition, an approach founded on genetic algorithm was reported in [10].

\section{Materials and methods}

Instead of using the real robot vehicle, we simulate the concept of robot vehicle movement represented by a nodal spot stirring in a known static indoor environment. The difficulty of finding the robot's path can be designated as a problem of steady-state heat transfer. The target is to be viewed in the analogy of heat transfer as a sink hauling heat in. The obstacles and borders of the environment are known as sources of heat and set at constant temperatures. The temperature distribution evolves through the heat conduction cycle, and the lines of heat flux circulating into the sink filling in the configuration space. This situation can be viewed as a communication mechanism between the target, obstacles, and the spots act as robots. The temperature distribution within the configuration space is then used as a steer for the robot to shift as of starting spot to the target spot by ensuing the heat stream from the highest to the lowest temperature point in the area. The environment temperature distribution is figured by engaging the function of harmonic to model the configuration space setup.

For the construction of the robot route, the domain $\Omega$ (denoted by $\partial \Omega$ ) consists of the external border walls, every obstacle in the configuration space, entire initial points, and target point. Arithmetically, a harmonic function in domain $\Omega \subset R^{n}$ is a function that fulfills Laplace's equation as shown below

$$
\nabla^{2} U=\sum_{i=1}^{n} \frac{\partial^{2} U}{\partial x_{i}^{2}}=0
$$

with

$$
\begin{aligned}
& x_{i}=\text { the } i \text {-th Cartesian coordinates } \\
& n=\text { the dimension }
\end{aligned}
$$


Laplace's equation can be efficiently solved with numerical technique such as Jacobi and Gauss-Seidel (GS), which are standard solutions for the problem. While Eq. (1) is solved in this paper using an accelerated iterative scheme for swifter computation.

The robot is characterized in this simulation by a nodal spot in the grid-shaped configuration space as shown in Fig. (1). Based on 5-point finite approximation from the configuration space, Fig. (2) shows a sample of its computational molecule, where $h$ is the distance between node points in each direction. Then, the numerical technique iteratively computes the function values for each node in order to satisfy Eq. (1). The uppermost temperature is allocated to the initial spot, however the target spot given as the lowest, while varied initial temperature values are consigned to the external borders wall and obstacles. There is no requirement to allocate initial temperature values to the starting spots. With Dirichlet boundary conditions, $U \mid \partial \Omega=c, c=$ constant, the solutions for Laplace's equation were examined. Upon gaining the potential values in the configuration space, it is possible to establish a smooth trajectory by outlining the temperature distribution with the steepest descent manner, where the algorithm monitors the negative gradient at the lowest temperature target spot from the beginning to successive points of lower temperature.

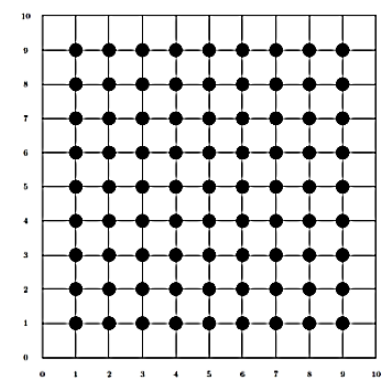

Fig. 1. Configuration space nodes in grid-shaped.

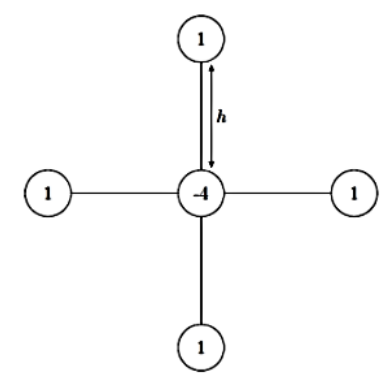

Fig. 2. Computational molecules of five-point approximation.

\subsection{Formulation of two-parameter over-relaxation iterative method}

From the robotics writings, the standard GS [3] and Successive Over-Relaxation (SOR) [11] were used as solutions for the problem (1). The solution of Laplace's equation in this analysis is computed by using a faster numerical solver, namely Two-parameter Over-Relaxation (TOR) iterative method. Consider the equation of the 2-dimensional Laplace's as set out in (1) be defined as 


$$
\frac{\partial^{2} U}{\partial x^{2}}+\frac{\partial^{2} U}{\partial y^{2}}=0
$$

The approximation of Eq. (2), as commonly described in the subsequent equation, can be simplified over 5-point second-order finite difference formula,

$$
U_{i-1, j}+U_{i+1, j}+U_{i, j-1}+U_{i, j+1}-4 U_{i, j}=0
$$

The iterative scheme of SOR can be formulated, by appending a weighted parameter $\omega$ to Eq. (3), as the following equation

$$
U_{i, j}^{(k+1)}=\frac{\omega}{4}\left[U_{i-1, j}^{(k+1)}+U_{i+1, j}^{(k)}+U_{i, j-1}^{(k+1)}+U_{i, j+1}^{(k)}\right]+(1-\omega) U_{i, j}^{(k)} .
$$

For the purpose of enhancement, families of over-relaxation namely the Accelerated OverRelaxation (AOR) iterative scheme are implemented by dividing the weighted parameter $\omega$ from Eq. (4) into two weighted parameters called $r$ and $\omega$. The scheme for the AOR method given as

$$
\begin{aligned}
U_{i, j}^{(k+1)}= & \frac{r}{4}\left[U_{i-1, j}^{(k+1)}-U_{i-1, j}^{(k)}+U_{i, j-1}^{(k+1)}-U_{i, j-1}^{(k)}\right] \\
& +\frac{\omega}{4}\left[U_{i-1, j}^{(k)}+U_{i+1, j}^{(k)}+U_{i, j-1}^{(k)}+U_{i, j+1}^{(k)}\right]+(1-\omega) U_{i, j}^{(k)}
\end{aligned} .
$$

The TOR iterative method, which is a generalization of the AOR method, is executed to boost up the convergence speed. The TOR scheme containing three different optimum relaxation parameters defined as $r, r^{\prime}$, and $\omega$ and written as

$$
\begin{aligned}
U_{i, j}^{(k+1)}= & \frac{r}{4} U_{i, j-1}^{(k+1)}+\frac{r^{\prime}}{4} U_{i-1, j}^{(k+1)}+\frac{\omega}{4}\left(U_{i, j+1}^{(k)}+U_{i+1, j}^{(k)}\right) \\
& +\left(\frac{\omega-r}{4}\right) U_{i, j-1}^{(k)}+\left(\frac{\omega-r^{\prime}}{4}\right) U_{i-1, j}^{(k)}+(1-\omega) U_{i, j}^{(k)}
\end{aligned} .
$$

The uncertain optimum values of all three $r, r^{\prime}$, and $\omega$, gave no restriction in getting the minimum iteration counts. Hadjidimos [12] specified that the values $r$ and $r^{\prime}$ are commonly selected to be as near as the value of $\omega$ for the corresponding SOR, where $1 \leq \omega<2$.

\section{Experiments and results}

In this study, the setup of the environments includes four different sizes: $300 \times 300,600 \times 600$, $900 \times 900$ and $1200 \times 200$. From Fig. 3, the target point is set at the lowest and fixed temperature values, although no specific temperature values are given to all three start spots. Various obstacles with different shapes have been placed in the environment. Dirichlet boundary condition is introduced in the initial setting, in which the obstacles and walls remain 
mounted at high temperatures. With the exception of the target spot at the lowest temperature value, all the other spots are set at zero value.

The development of computation is performed on AMD A10-7400P Radeon R6, 10 Compute Cores $4 \mathrm{C}+6 \mathrm{G}$ running at $2.50 \mathrm{GHz}$ speed with $8 \mathrm{~GB}$ of RAM. The iteration progression for numerically measuring temperature values at each point continues until the circumstance of stoppage is encountered. If the temperature values show no more changes, the loop will end and the variance of computation values should be diminutive, i.e. 1.0 $0^{-15}$. This overhead precision is required in the solutions to avert the development of a flat area, better known as saddle points, from failing the generation of paths.

Tables 1 and 2 display the iteration counts and time taken, respectively, required to measure every temperature values in the environment for each numerical approaches compared in this experiment. Evidently, the TOR iterative method is verified to give the best performance when compared with the SOR and AOR method.

Table 1. Performance of the proposed methods in terms of iteration count.

\begin{tabular}{|l|c|cccc|}
\cline { 3 - 6 } \multicolumn{1}{c|}{} & \multirow{2}{*}{ Methods } & \multicolumn{4}{|c|}{$\mathbf{N \times \mathbf { N }}$} \\
\cline { 3 - 6 } Case 1 & & $\mathbf{3 0 0}$ & $\mathbf{6 0 0}$ & $\mathbf{9 0 0}$ & $\mathbf{1 2 0 0}$ \\
\hline \multirow{4}{*}{ Case 2 } & SOR & 2228 & 8776 & 19254 & 33558 \\
& AOR & 2006 & 7973 & 17538 & 30573 \\
& TOR & 1893 & 7553 & 16642 & 29008 \\
\hline \multirow{4}{*}{ Case 3 } & SOR & 3624 & 14644 & 33004 & 57484 \\
& AOR & 3236 & 13165 & 29680 & 51738 \\
& TOR & 2843 & 11685 & 26393 & 46021 \\
\hline & SOR & 2507 & 9868 & 21654 & 37762 \\
& AOR & 2288 & 9025 & 19840 & 34601 \\
& TOR & 2067 & 8217 & 18052 & 31519 \\
\hline
\end{tabular}

Table 2. Performance of the proposed methods in terms of execution time (in seconds).

\begin{tabular}{|c|c|cccc|}
\cline { 3 - 6 } \multicolumn{1}{c|}{} & \multirow{2}{*}{ Methods } & \multicolumn{4}{|c|}{$\mathbf{N \times \mathbf { N }}$} \\
\cline { 3 - 6 } Case 1 & SOR & 10.69 & 251.72 & 1270.23 & 4077.22 \\
& AOR & 10.27 & 248.24 & 1226.66 & 3976.33 \\
& TOR & 9.39 & 233.83 & 1194.50 & 3732.02 \\
\hline \multirow{4}{*}{ Case 2 } & SOR & 16.22 & 427.27 & 2190.45 & 7432.68 \\
& AOR & 18.66 & 418.45 & 2073.25 & 7254.02 \\
& TOR & 15.20 & 369.55 & 1927.30 & 6300.13 \\
\hline \multirow{4}{*}{ Case 3 } & SOR & 11.02 & 281.85 & 1441.47 & 4853.57 \\
& AOR & 12.52 & 281.78 & 1423.54 & 4743.21 \\
& TOR & 10.91 & 255.82 & 1292.23 & 4269.42 \\
\hline
\end{tabular}

\section{Discussion}

As soon as the temperature values have been reached, the route is created by executing the steepest descent search commencing from the initial to the target spot. The route generation process is very fast, in which the algorithm basically selects the lowest temperature value of its adjacent points from the current point. This practice goes on until the target spot has been reached. Fig. 3 illustrates that the routes were positively generated in an obstacle setting build the temperature distribution profile gained by numerical computation. Every starting spot (square/green point) successfully connected to the specified target spot (round/red point) and escaped all different obstacles that are set in the environment. 


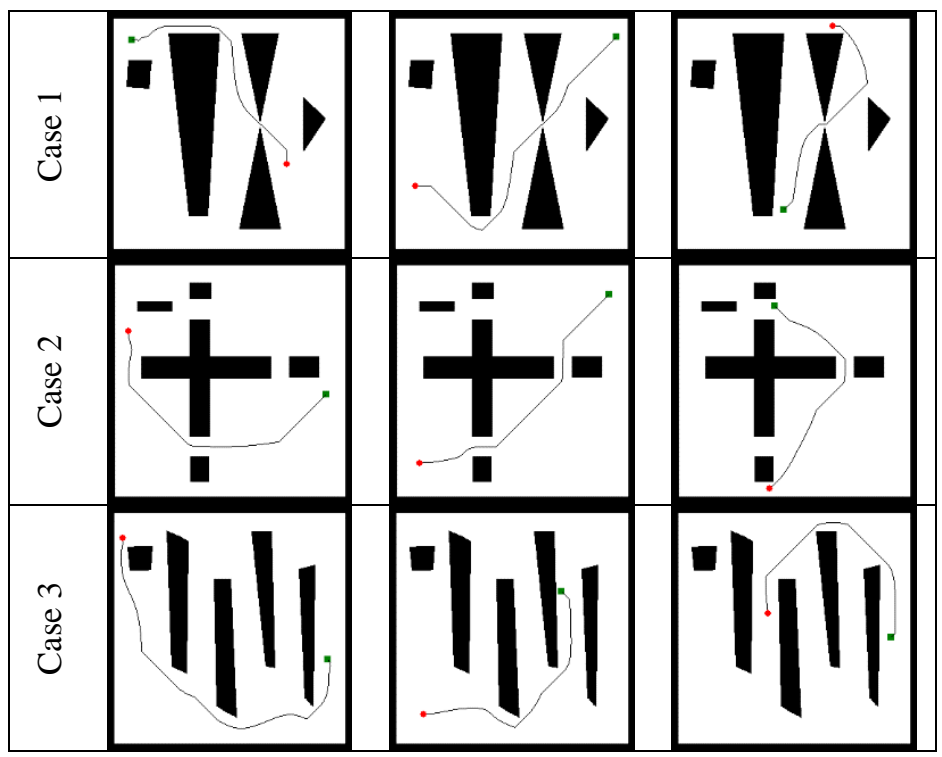

Fig. 3. The paths generated from different starting position (green dot/square dot) and target (red $\operatorname{dot} /$ circle dot) positions for various environment using proposed method.

\section{Conclusion}

This experiment reveals that the solution of robot pathfinding problems via numerical approaches is in fact very smart and practicable, by reason of the recent advancements and newly emerging techniques, along with the accessibility of fast machines in present day. In comparison to the standard SOR and AOR method, as presented in the result tables, the TOR iterative method is verified to give the best performance. An increment to the number of obstacles does not negatively affect the results, as computations are actually getting more rapid as the engaged zones by obstacles are disregarded in the computational processes. The advantage of the developed algorithm is that, regardless of obstacles in their shape and position, the robot can always travel from the start to the target point, not only safely, but also on the optimal path. The results of this paper can essentially be categorized as a family of full-sweep iterations. In addition to the concept of full-sweep iteration, advance research on half-sweep $[13,14]$ and quarter-sweep [15] iterations will improve the convergence rate of the suggested standard iterative methods.

This research was supported by National Defence University of Malaysia for the funding of this article. The researchers declare that there is no conflict of interest regarding the publication of this study.

\section{References}

1. C.I. Connolly, R. Gruppen, J. Robotic Syst. 10(7), 931 - 946 (1993)

2. O. Khatib, IEEE T. Robotics Autom. 1, 500 - 505 (1985) 
3. C.I. Connolly, J.B. Burns, R. Weiss, Path planning using Laplace's equation, in Proceedings - IEEE International Conference on Robotics and Automation, 13-18 May 1990, Cincinnati, OH, USA (1990)

4. S. Akishita, S. Kawamura, K. Hayashi, Japan-USA Sym. Flex. Autom.: A Pacif. Rim Conf., 139 - 142 (1990)

5. A. Saudi, J. Sulaiman, Int. J. Imag. Robot. 17(3), 77 - 90 (2017)

6. A.A. Dahalan, J. Sulaiman, M.S. Muthuvalu, AIP Conf. Proc. 1544, 116 (2013)

7. A.R. Willms, X.Y, Simon, IEEE T. Syst. Man Cy. B. 38(3) (2008)

8. G.E. Jan, K.Y. Chang, I. Parberry, IEEE/ASME T. Mechatronics 13(4), 451 - 460 (2008)

9. P. Bhattacharya, M.L. Gavrilova, IEEE Robot. Autom. Mag. 15(2), 58 - 66 (2008)

10. Y. Liang, L. Xu, Global path planning for mobile robot based genetic algorithm and modified simulated annealing algorithm, in Proceedings of the 1st ACM/SIGEVO Summit on Genetic and Evolutionary Computation, GEC'09, June 2009, Shanghai, China (2009)

11. A. Saudi, J. Sulaiman, M.H.A. Hijazi, Res. J. Appl. Sci. 9(6), 354 - 360 (2014)

12. A. Hadjidimos, Math. Comput. 32(141), 149 - 157 (1978)

13. A.A. Dahalan, J. Sulaiman, Appl. Math. Sci. 10(2), 45 - 57 (2016)

14. A.A. Dahalan, N.A. Shattar, J. Sulaiman, J. Eng. Appl. Sci. 11(9), 1891 - 1897 (2016)

15. A.A. Dahalan, J. Sulaiman, Int. J. Math. Anal. 35(9), 1733 - 1746 (2015) 